\title{
Glycyrrhizin Alleviates Nonalcoholic Steatohepatitis via Modulating Bile Acids and Meta-Inflammation ${ }^{\mathbb{S}}$
}

\author{
Tingting Yan, Hong Wang, Lijuan Cao, Qiong Wang, Shogo Takahashi, Tomoki Yagai, Guolin Li, \\ Kristopher W. Krausz, Guangji Wang, Frank J. Gonzalez, and Haiping Hao
}

State Key Laboratory of Natural Medicines, Key Laboratory of Drug Metabolism and Pharmacokinetics, China Pharmaceutical University, Nanjing, Jiangsu, China (Ti. Y., H.W., L.C., G.W., H.H.); and Laboratory of Metabolism, Center for Cancer Research, National Institutes of Health National Cancer Institute, Bethesda, Maryland (Ti. Y., Q.W., S.T., To.Y., G.L., K.W.K., F.J.G.)

Received April 12, 2018; accepted June 27, 2018

\section{ABSTRACT}

Nonalcoholic steatohepatitis (NASH) is the progressive stage of nonalcoholic fatty liver disease that may ultimately lead to cirrhosis and liver cancer, and there are few therapeutic options for its treatment. Glycyrrhizin (GL), extracted from the traditional Chinese medicine liquorice, has potent hepatoprotective effects in both preclinical animal models and in humans. However, little is currently known about its effects and mechanisms in treating NASH. To explore the effects of GL on NASH, GL or its active metabolite glycyrrhetinic acid (GA) was administered to mice treated with a methionine- and choline-deficient (MCD) diet-induced NASH model, and histologic and biochemical analyses were used to measure the degree of lipid disruption, liver inflammation, and fibrosis. GL significantly improved MCD diet-induced hepatic steatosis, inflammation, and fibrosis and inhibited activation of the NLR family pyrin domain-containing 3 (NLRP3) inflammasome. GL significantly attenuated serum bile acid accumulation in MCD diet-fed mice partially by restoring inflammation-mediated hepatic farnesoid $X$ receptor inhibition. In Raw 264.7 macrophage cells, both GL and GA inhibited deoxycholic acid-induced NLRP3 inflammasome-associated inflammation. Notably, both intraperitoneal injection of GL's active metabolite GA and oral administration of GL prevented NASH in mice, indicating that GL may attenuate NASH via its active metabolite GA. These results reveal that GL, via restoration of bile acid homeostasis and inhibition of inflammatory injury, can be a therapeutic option for treatment of NASH.

\section{Introduction}

Nonalcoholic fatty liver disease (NAFLD) affects $25 \%$ of the global adult population and has become a leading cause of chronic liver disease in Western countries and increasingly affects the Asian population (Younossi et al., 2016). Early-stage NAFLD is asymptomatic; however, when NAFLD progresses to nonalcoholic steatohepatitis (NASH), patients have a high risk of adverse events including fibrosis, cirrhosis, and NASH-driven hepatocellular carcinoma (Wong et al., 2014, 2016; Zoller and Tilg, 2016). Traditional Chinese medicine has been investigated for treating NASH (Jadeja et al., 2014; Zhang and Schuppan, 2014). Glycyrrhizin (GL) is a potent hepatoprotective constituent extracted from the traditional Chinese medicine liquorice, as revealed by various animal experimental liver injury

This work was supported by the National Natural Science Foundation of China [Grants 81430091, 81720108032, 81421005, 91429308, and 81603194], the Project for Major New Drug Innovation and Development [Grant 2015ZX09501010], the Overseas Expertise Introduction Project for Discipline Innovation [Grant G20582017001], the China Postdoctoral Science Foundation [Grants 2016M600455 and 2017T100423], and the Intramural Research Program of the National Institutes of Health National Cancer Institute.

https://doi.org/10.1124/dmd.118.082008.

S This article has supplemental material available at dmd.aspetjournals.org. models (Li et al., 2014; Yan et al., 2016), and thus is clinically used for treating chronic liver diseases in some Asian countries (Li et al., 2014). Clinical prescription preference for GL is found mainly for treating chronic liver diseases, especially for viral hepatitis in China and Japan ( $\mathrm{Li}$ et al., 2014), whereas preclinical studies of GL in treating liver diseases have mostly focused on viral hepatitis and drug/toxin-induced hepatotoxicity ( $\mathrm{Li}$ et al., 2014; Yan et al., 2016; Zhou et al., 2016). However, the effect of GL in preventing NASH is largely unknown. Thus far, only glycyrrhetinic acid (GA), the metabolite of GL in vivo, has been shown to prevent high-fat dietinduced NAFLD in rats in vivo and free fatty acid-induced toxicity in HepG2 cells in vitro (Wu et al., 2008). Whether and how GL and GA affect NASH (the progressive stage of NAFLD) is still unknown.

Bile acid homeostasis, which is mainly modulated by the farnesoid $\mathrm{X}$ receptor (FXR), is disrupted in experimental NASH mouse models and in clinical human NASH patients (Arab et al., 2017). Dysregulated bile acid disruption correlates with nonalcoholic steatosis scores in obese NAFLD (Bechmann et al., 2013), causes cholestatic liver injury (Schoemaker et al., 2003), and promotes liver carcinogenesis in a NASH-driven hepatocellular carcinoma model in mice (Xie et al., 2016). Furthermore, a bile acid sequestrant was found to prevent NAFLD and NASH (Arab et al., 2017), suggesting that suppressing the accumulation of bile acids during NASH could benefit NASH treatment. On the other hand, the NLR family pyrin

ABBREVIATIONS: ACTD, actinomycin D; ALT, alanine aminotransferase; AST, aspartate aminotransferase; CA, cholic acid; CASP, caspase; DAMP, damage-associated molecular pattern; DCA, deoxycholic acid; FACS, fluorescence-activated cell sorting; FXR, farnesoid X receptor; GA, glycyrrhetinic acid; GL, glycyrrhizin; GW4064, 3-[2-[2-chloro-4-[[3-(2,6-dichlorophenyl)-5-(1-methylethyl)-4-isoxazolyl]methoxy]phenyl]ethenyl]benzoic acid; IL, interleukin; LPS, lipopolysaccharide; MCA, muricholic acid; MCC950, N-[[(1,2,3,5,6,7-hexahydro-s-indacen-4-yl)amino]carbonyl]-4-(1-hydroxy1-methylethyl)-2-furansulfonamide sodium salt; MCD, methionine- and choline-deficient; MCS, methionine- and choline-sufficient; NAFLD, nonalcoholic fatty liver disease; NASH, nonalcoholic steatohepatitis; NF- $\kappa \mathrm{B}$, nuclear factor $\kappa \mathrm{B}$; SHP, small heterodimer partner; SREPB, sterol regulatory element-binding transcription factor; T, tauro; TC, total cholesterol; TG, triglyceride; TLR, Toll-like receptor; TNF, tumor necrosis factor. 
domain-containing 3 (NLRP3) inflammasome is known to be required for hepatocyte pyroptosis, liver inflammation, and fibrosis development in NAFLD (Wree et al., 2014a,b). Metabolic homeostasis is associated with the immune system (Osborn and Olefsky, 2012), among which several bile acids were recently demonstrated to directly activate the NLRP3 inflammasome (Hao et al., 2017), providing a link between immune dysfunction and metabolic disorder. Since bile acids accumulate in NASH livers, it is reasonable to infer that toxic bile acids induce NLRP3 inflammasome activation and could be a key factor in mediating NASH progression. The bile acid-NLRP3 inflammasome axis is a potential pharmacological target for treating NASH.

GL was demonstrated to inhibit lithocholic acid-induced cholestatic liver injury (Wang et al., 2012), bile acid-induced cytotoxicity in rat hepatocytes (Gumpricht et al., 2005), and $\alpha$-naphthyl isothiocyanate-induced liver injury and bile acid disruption (Wang et al., 2017), indicating a potential role for GL in treating cholestatic liver injury. In addition, GL is also an inhibitor of NLRP3 inflammasome activation in macrophages in vitro and in NLRP3 inflammasome-associated adipose tissue inflammation in mice (Honda et al., 2014). Thus, GL might target the bile acid-NLRP3 inflammasome to elicit its hepatoprotective function for decreasing NASH.

In this study, the methionine- and choline-deficient (MCD) diet-induced NASH mouse model was used to examine the hepatoprotective effect and potential mechanisms of GL. GL was found to potently inhibit MCD diet-induced liver lipid accumulation, inflammation, and fibrosis. GL dampened activation of the NLPR3 inflammasome and restored bile acid homeostasis. Moreover, GL also directly inhibited deoxycholic acid-induced NLRP3 inflammasome activation. GL gavage as well as GA significantly prevented MCD diet-induced liver injury, suggesting that GL by oral intake might be applied in the clinic as a therapeutic option for NASH patients.

\section{Materials and Methods}

Chemicals and Reagents. GL (97\%) was purchased from TCI (Shanghai, China). 18 $\beta$-GA (97\%), actinomycin D (ACTD), Tween 80, and lipopolysaccharides (LPSs) from Escherichia coli O111:B4 were purchased from Sigma (St. Louis, MO). Recombinant human tumor necrosis factor (TNF)- $\alpha$ was purchased from R\&D Systems (catalog number 210-TA; Minneapolis, MN). The MCD diet and the methionine- and choline-sufficient (MCS) diet were purchased from Trophic Animal Feed High-Tech Co. Ltd. (Nantong, China) or Dyets Inc. (Bethlehem, PA). The In Situ Cell Death Detection Kit was purchased from Roche (Indianapolis, IN).

Experimental Animals and Treatments. Six- to 8-week-old male C57BL/6 mice were obtained from the Academy of Military Medical Sciences (Beijing, China) or the National Institutes of Health (Bethesda, MD). Mice were acclimatized to the facilities for a week and then randomly divided into different groups. The animal room was maintained at $25 \pm 2{ }^{\circ} \mathrm{C}$ with a 12-hour/12-hour light/dark cycle and $50 \% \pm 10 \%$ humidity. All animal studies were approved by the Animal Ethics Committee of China Pharmaceutical University and carried out in accordance with the National Institutes of Health Guide for the Care and Use of Laboratory Animals.

To assess the influence of GL in treating NASH, mice were randomly divided into four groups with different treatments for 8 weeks: the MCS group (saline, i.p.), the MCS+GL50 group (50 $\mathrm{mg} / \mathrm{kg}$ GL per day, i.p.), the MCD group (saline, i.p.), and the MCD+GL50 group (50 mg/kg GL per day, i.p.). All animals were fed the MCD or MCS diet for 6 weeks and then injected with $50 \mathrm{mg} / \mathrm{kg}$ GL per day for 2 consecutive weeks, while mice were fed the matched MCD diet or MCS diet for the last 2 weeks until the end of the experiments. All mice were fasted for 4 hours with free access to water before killing. To test the effects of GA injection in preventing NASH, mice were divided into three groups with different treatments for 6 weeks: the MCS group (MCS diet feeding, saline, i.p.), the MCD group (MCD diet feeding, 5\% Tween 80/saline, i.p.), and the MCD+GA30 group (MCD diet feeding, $30 \mathrm{mg} / \mathrm{kg}$ GA per day, dissolved in $5 \%$ Tween $80 /$ saline, i.p.). Similarly, in the study to test the effect of GL gavage, three groups of mice were treated accordingly: the MCS group (MCS diet feeding, saline, i.p.), the MCD group (MCD diet feeding, saline, p.o.), and the MCD+GL50 group (MCD diet feeding, $50 \mathrm{mg} / \mathrm{kg}$ GL per day, p.o.). All mice were fed the MCD or MCS diet for 6 weeks and injected with control vehicle or $50 \mathrm{mg} / \mathrm{kg}$ GL per day gavage for the last 3 consecutive weeks, respectively. All mice were killed with $\mathrm{CO}_{2}$ asphyxiation, and blood and liver tissues were collected and stored for further use. GL was dissolved in saline and the $\mathrm{pH}$ value was adjusted to $7.0-7.5$ with $1 \mathrm{M}$ sodium hydroxide solution (Sigma). GA was freshly dissolved in saline containing 5\% Tween 80.

Histology Analysis. Formalin-fixed liver tissues were embedded in paraffin and $5-\mu$ m-thick sections were cut for hematoxylin and eosin staining, terminal deoxynucleotidyl transferase-mediated digoxigenin-deoxyuridine nick-end labeling staining, and Masson trichrome staining based on the respective manual protocol. A portion of the fresh liver was embedded in Tissue-Tek Optimal Cutting Temperature compound (Sakura Finetek, Torrance, CA) and then flash frozen for Oil Red O staining. Further sample processing and analysis was performed in the Department of Pathophysiology at the Affiliated Hospital of Nanjing University of Chinese Medicine (Nanjing, Jiangsu, China; Figs. 1-3) and at Histoserv Inc. (Germantown, MD; Fig. 7).

Targeted Bile Acid Metabolome Analysis. Levels of main bile acid species were determined by high-performance liquid chromatography quadrupole timeof-flight tandem mass spectrometry as described previously (Hao et al., 2017).

In Vitro Cell Culture and Treatment. Human HepG2 cells were obtained from American Type Culture Collection (Manassas, VA). To test the protective effects of GL or GA on ACTD/TNF- $\alpha$-induced HepG2 cell death, HepG2 cells were seeded in 96-well plates for cell viability assays or six-well plates for fluorescence-activated cell sorting (FACS) assays and then grown to $70 \%-90 \%$ confluence before use. HepG2 cells were pretreated with control dimethylsulfoxide, GL, or GA together with $0.3 \mu \mathrm{M}$ ACTD for 30 minutes and then treated with recombinant human TNF- $\alpha$ for an additional 13 or 24 hours for cell viability assays with the Cell Counting Kit-8 (Dojindo Laboratories, Kumamoto, Japan). For FACS assays, samples were collected at 13 hours after treatment and analyzed according to the manufacturer's instructions for the Annexin $\mathrm{V}$ fluorescein isothiocyanate apoptosis detection kit (BD Biosciences, San Diego, CA).

Reporter Luciferase Assay. The PGL4-small heterodimer partner (Shp)-TK firefly luciferase construct and human FXR expression plasmid was provided by Grace L. Guo (Rutgers University, New Brunswick, NJ). A Renilla luciferase reporter gene (pRL-luciferase; Promega, Madison, WI) was used as a transfection efficiency control. The plasmids were transfected into HepG2 cells that were cultured in 10\% RPMI 1640 medium and seeded in 24-well plates at 70\%-90\% confluence. Cells were transfected with plasmids using Lipofectamine 3000 reagent (Invitrogen/Thermo Fisher Scientific, Waltham, MA) for 24 hours and then treated with LPS, GL, or GA for another 24 hours. The cells were lysed and luciferase activities were measured with the Dual Luciferase Reporter Assay kit (Promega) and a Genios Pro luminescence plate reader (Tecan, Research Triangle Park, NC).

Serum Biochemical Analysis. Biochemical parameters, including levels of alanine aminotransferase (ALT), aspartate aminotransferase (AST), total cholesterol (TC), triglyceride (TG), low-density lipoprotein cholesterol, and highdensity lipoprotein cholesterol in serum and TC and TG in the liver, were quantified using a standard clinical automatic analyzer at Zhongda Hospital, Affiliated Hospital of Southeastern University (Jiangsu, China).

Western Blot Analysis. Liver samples were lysed in radioimmunoprecipitation assay lysis buffer (Millipore, Temecula, CA) with protease and phosphatase inhibitors (Halt Protease and Phosphatase Inhibitor Cocktail, catalog number 78446; Thermo Scientific, Rockford, IL), and the protein extracts were then separated by SDS-PAGE and transferred to a polyvinylidene difluoride membrane. The membrane was incubated overnight at $4^{\circ} \mathrm{C}$ with antibodies against mouse $\beta$-actin (catalog number 4970; Cell Signaling Technology, Danvers, MA), mouse monoclonal anti-caspase 1 (CASP1; catalog number sc-56036; Santa Cruz Biotechnology, Dallas, TX), and rabbit polyclonal anti-interleukin-1 $\beta$ (IL-1 $\beta$; catalog number sc-7884; Santa Cruz Biotechnology).

Enzyme-Linked Immunosorbent Assay of Serum IL-1 $\beta$ Levels in Mice. An IL- $1 \beta$ enzyme-linked immunosorbent assay kit was purchased from R\&D Systems (catalog number MLB00C). Fifty microliters of serum was used for analysis based on the manual protocol accordingly.

Quantitative Polymerase Chain Reaction. Total tissue RNA extraction was performed by using RNAiso Plus reagent (Takara, Biotechnology Co. Ltd., Dalian, China). Purified total RNA was reverse transcribed using the Prime Script RT Reagent Kit (Takara Biotechnology Co. Ltd.). Quantitative polymerase chain reaction was performed by using the ABI PRISM 7000 Sequence Detection System (Applied Biosystems, Bedford, MA) and the SYBR Green reagent kit (Bio-Rad Co. Ltd., Shanghai, China). Values were normalized to glyceraldehyde 3-phosphate dehydrogenase. Sequences for quantitative polymerase chain reaction primers are available upon request.

Statistical Analysis. Data are presented as means \pm S.D. Statistical differences between experimental groups were determined with the two-tailed $t$ test or 
A

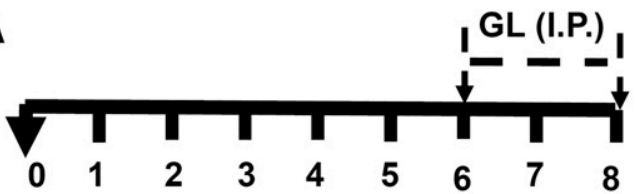

\section{Matched MCS or MCD Feeding Time (W, Week)}

B

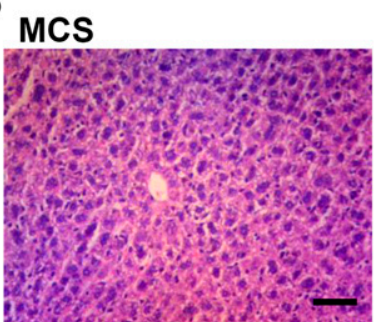

MCD

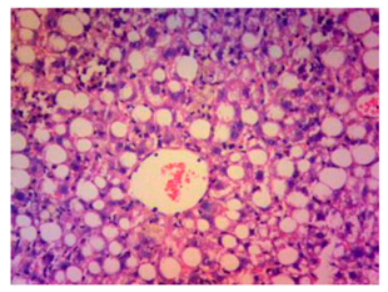

MCS+GL50

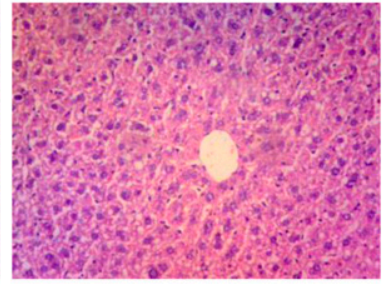

$M C D+G L 50$

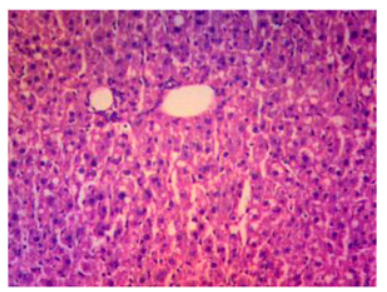

C

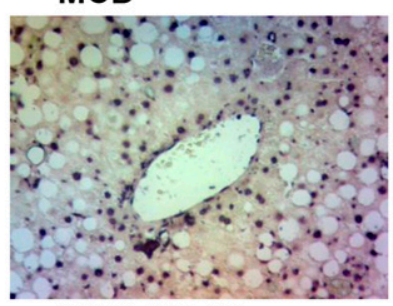

MCD+GL50

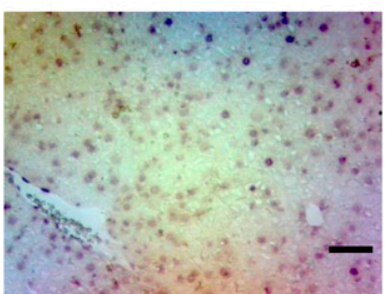

Fig. 1. GL significantly alleviates MCD dietinduced liver damage, improves TUNEL staining, and reduces serum transaminases. (A) Mouse experiment procedure scheme. (B) $\mathrm{H} \& \mathrm{E}$ staining of liver sections. (C) TUNEL staining analysis of paraffin-embedded livers. (D) Serum ALT levels. (E-G) NAFLD scoring statistics for $\mathrm{H} \& \mathrm{E}$ slides for NAFLD activity (E), steatosis scores (F), and inflammation scores (G). Data are presented as means \pm S.D. Statistical differences between experimental groups were determined by the two-tailed $t$ test ( $n=6-8$ for each group). $* * P<0.01$; ${ }^{*} * * P<0.001$ vs. the MCD group; ${ }^{\# \# \#} P<$ 0.001 vs. the MCS group. H\&E, hematoxylin and eosin; TUNEL, terminal deoxynucleotidyl transferase-mediated digoxigenin-deoxyuridine nick-end labeling. Original magnification, $\times 20$. Scale bar, $50 \mu \mathrm{m}$.
D

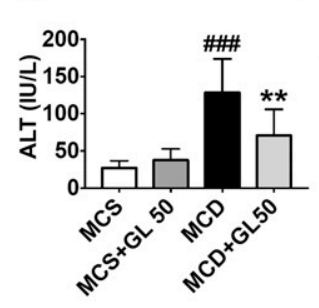

$\mathrm{E}$

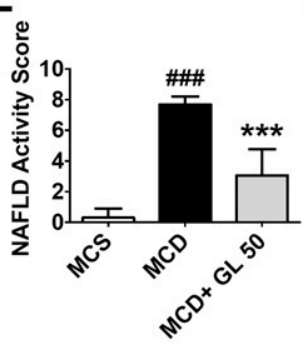

$\mathbf{F}$

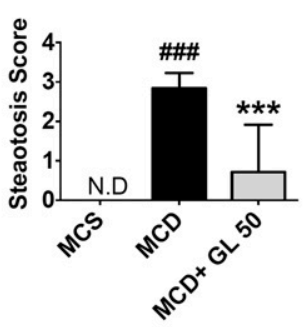

G

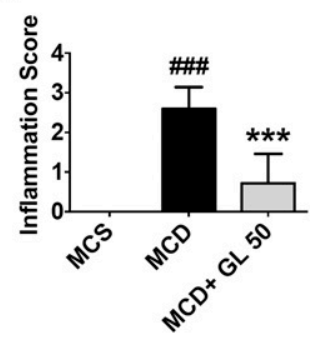

one-way analysis of variance followed by the Dunnett multiple-comparisons test in GraphPad Prism 7.0 software (GraphPad Software Inc., La Jolla, CA) as stated. $P$ values less than 0.05 were considered statistically significant.

\section{Results}

GL Attenuates NASH-Induced Liver Injury. To determine whether GL attenuates MCD diet-induced liver injury in vivo, C57BL/6 mice were treated with GL after MCD diet feeding for 6 weeks, and the GL treatment continued for the last 2 weeks while the mice were fed the matched MCD diet or the MCS diet during the injection (Fig. 1A). GL treatment did not significantly affect food intake and body weight (Supplemental Fig. 1, A and B) or the liver index (Supplemental Fig. 1C) in both MCS and MCD diet-fed mice. Hematoxylin and eosin staining data showed that $50 \mathrm{mg} / \mathrm{kg}$ GL sharply decreased hepatic steatosis in MCD diet-fed mice, whereas $50 \mathrm{mg} / \mathrm{kg}$ GL treatment alone did not cause liver toxicity (Fig. 1B). Apoptotic hepatocyte death is known to be involved in NASH (Feldstein et al., 2003); therefore, liver tissues were analyzed by terminal deoxynucleotidyl transferase-mediated digoxigenin-deoxyuridine nick-end labeling staining to measure apoptosis. GL treatment significantly reduced the positive staining compared with control saline treatment in MCD diet-fed mice (Fig. 1C). Consistently, the apoptosis marker gene mRNA, B cell leukemia/lymphoma 2 related protein A1c (Bcl2alc) Bcl2alc, was also significantly inhibited by GL treatment in MCD diet-fed mice (Supplemental Fig. 1D). Since TNF- $\alpha$ induces apoptosis (Wang et al., 1996) and ACTD/TNF- $\alpha$ is frequently used to mimic apoptotic cell death (Leist et al., 1994), the effects of GL in alleviating ACTD/TNF- $\alpha$-induced apoptosis were examined in HepG2 cells. GL directly inhibited an ACTD/ TNF- $\alpha$-induced decrease in cell viability at both 13 and 24 hours after ACTD/TNF- $\alpha$ treatment (Supplemental Fig. 1, E and F) and ameliorated the increase in apoptotic cell death as assessed by the FACS assay (Supplemental Fig. 1G), supporting that GL could directly inhibit TNF- $\alpha$-mediated apoptosis. In addition, serum ALT levels were increased in the MCD dietfed group but were markedly decreased in the MCD+GL50-treated group (Fig. 1D). NAFLD scores among the MCS, MCD, and MCD+GL50 groups by a double-blinded analysis also showed that the MCD diet induced a significant increase in NAFLD activity, steatosis, and inflammation in livers of the MCD group, which were all markedly alleviated by GL treatment (Fig. $1, \mathrm{E}-\mathrm{G})$. Taken together, these results suggest that GL rescues apoptotic liver injury induced by MCD diet feeding, at least partially through directly inhibition of TNF- $\alpha$-induced hepatocyte apoptosis.

GL Improves NASH-Related Liver Fibrosis. Liver fibrosis is a hallmark of NAFLD progression to NASH. Masson trichrome staining of collagen was used to measure liver fibrosis, and GL was found to significantly ameliorate NASH-associated collagen deposition induced by MCD diet feeding (Fig. 2A). $\alpha$-Smooth muscle actin is another 
A
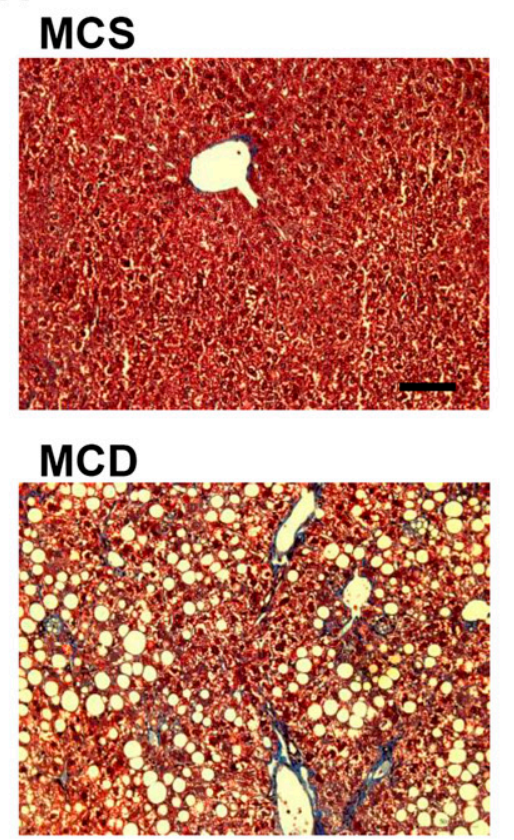

C
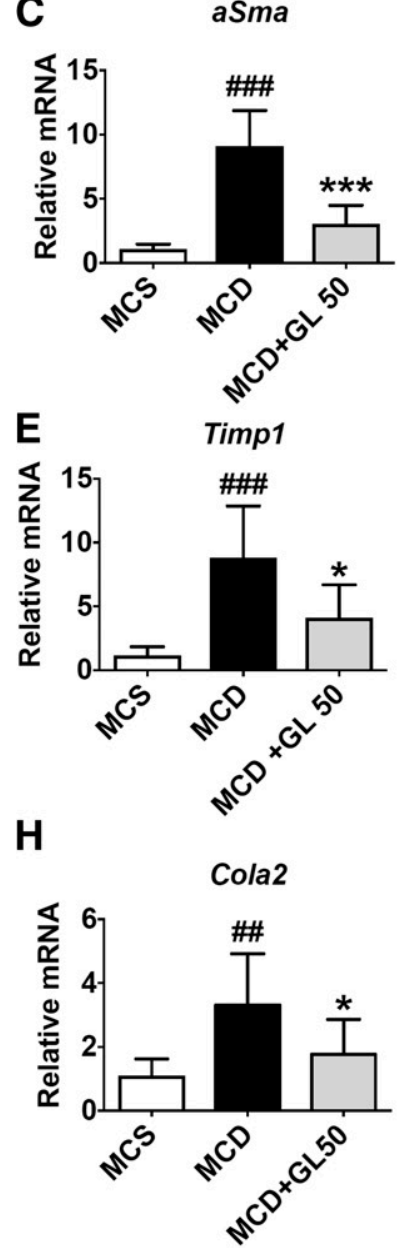

\section{MCS+GL50}

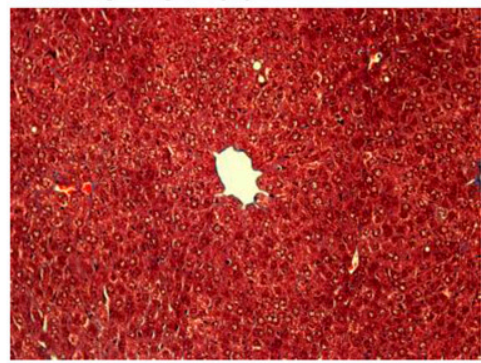

MCD+GL50

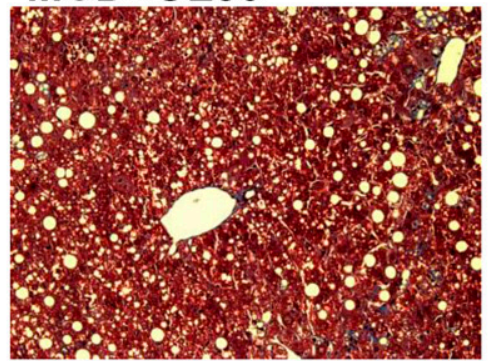

D

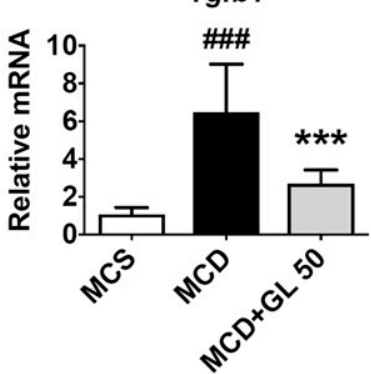

B

\section{MCS}

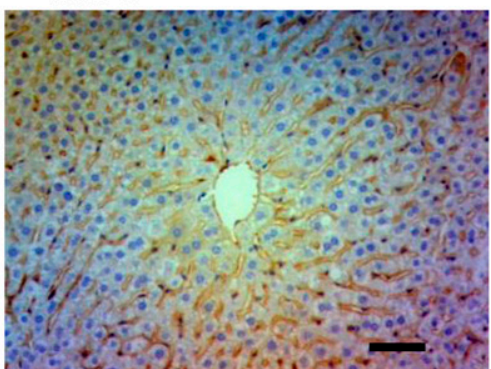

MCD

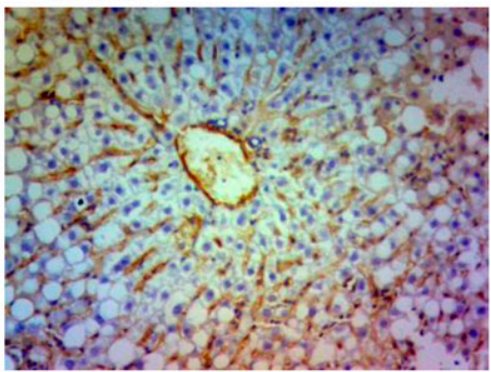

MCD + GL50

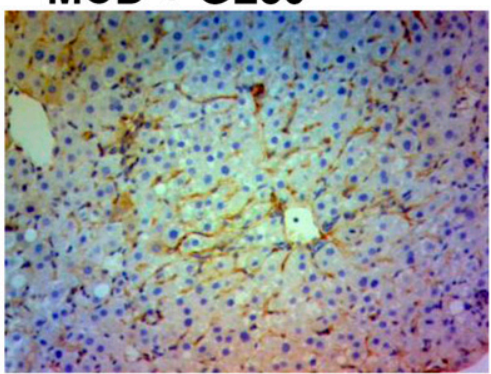

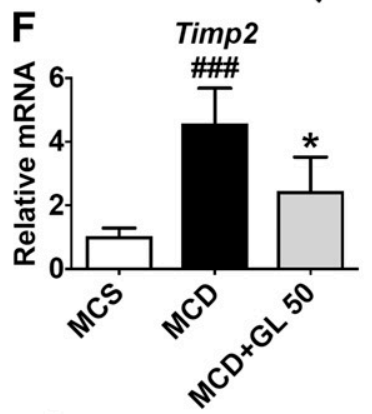

G

Cola1
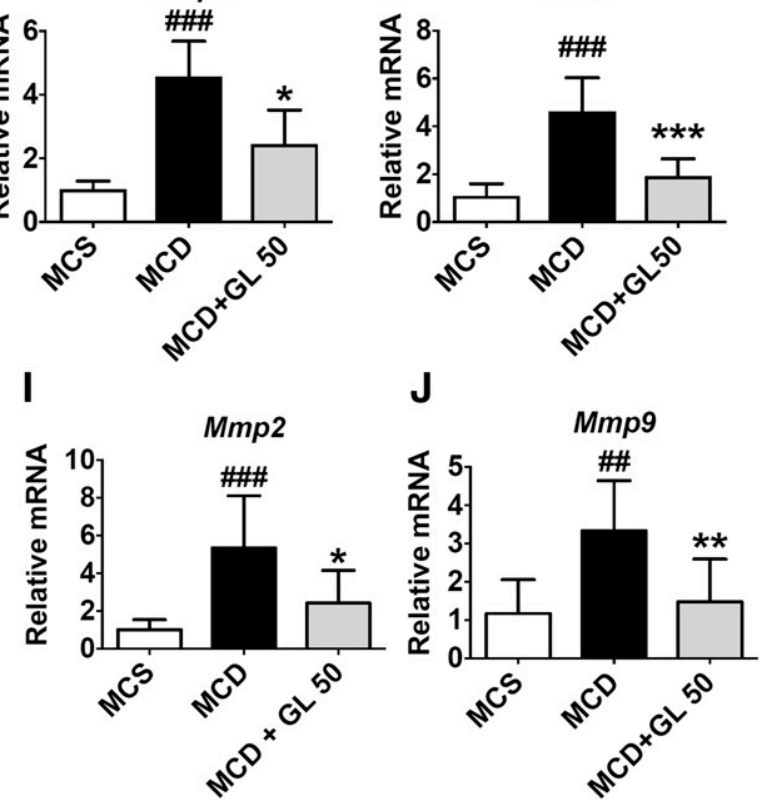

Fig. 2. GL significantly dampens MCD-induced liver fibrogenesis. (A and B) Masson trichrome staining (A) and immunohistochemistry staining of $\alpha$-SMA (B) for paraffinembedded livers. (C-F) Levels of $\alpha \operatorname{Sma}$ (C), Tgfb1 (D), Timp1 (E), and Timp2 (F) mRNAs in the liver. (G-J) Levels of Cola1 (G), Cola2 (H), Mmp2 (I), and Mmp9 (J) mRNAs in the liver. Data are presented as means \pm S.D. Statistical differences between experimental groups were determined by the two-tailed $t$ test $(n=6-8$ in each group). $* P<0.05 ; * * P<0.01 ; * * * P<0.001$ vs. the MCD group; ${ }^{\# \# P}<0.01 ;{ }^{\# \# \#} P<0.001$ vs. the MCS group. Cola, collagen; Mmp, matrix metalloproteinase; Sma, smooth muscle actin; Tgfbl, transforming growth factor $\beta$-1; Timp, tissue inhibitor of metalloproteinases. Original magnification, $\times 20$. Scale bar, $50 \mu \mathrm{m}$. 
A

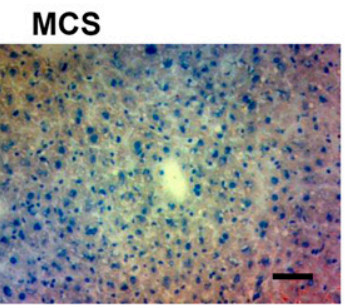

B

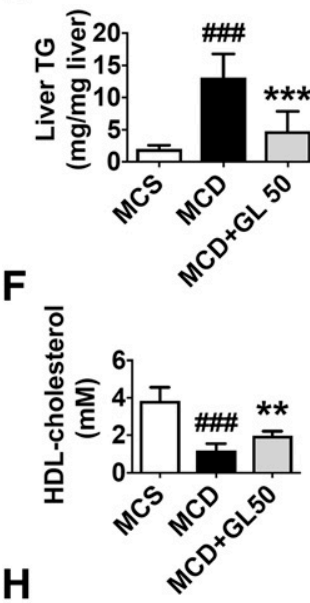

C

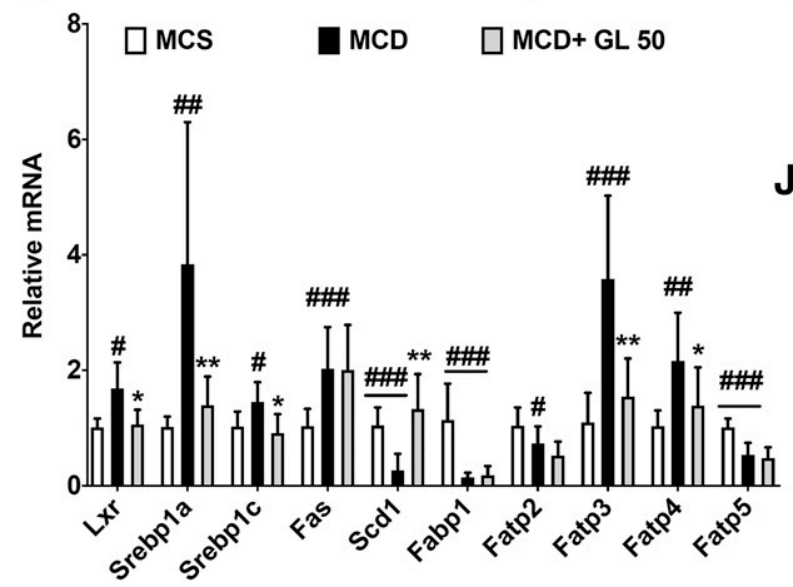

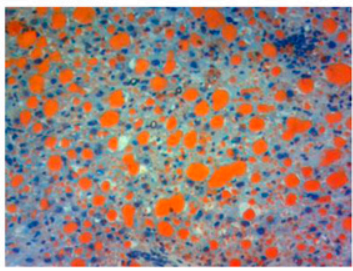

D
MCD
MCD+GL50

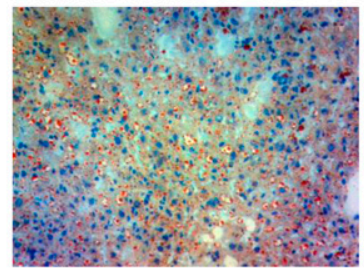

E

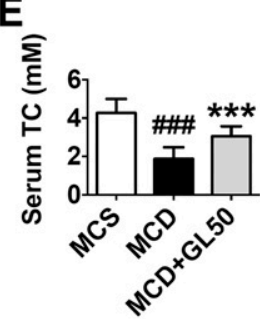

I

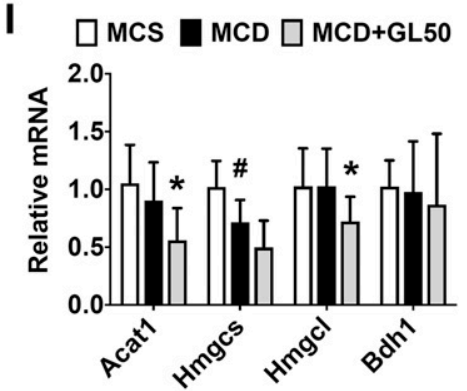

$\mathbf{J}$

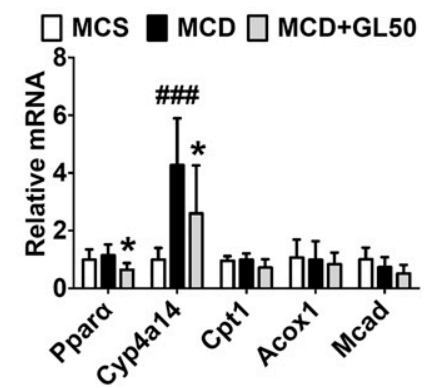

Fig. 3. GL significantly reduces MCD-induced lipid accumulation in the liver via inhibiting fatty acid uptake. (A) Oil Red $\mathrm{O}$ staining of liver sections. (B and C) Levels of liver TG (B) and TC (C). (D and E) Levels of serum TG (D) and TC (E). (F and G) Levels of serum HDL cholesterol (F) and LDL cholesterol (G). (H-J) Effect of GL in the mRNA expression involved in the pathway of lipogenesis $(\mathrm{H})$, ketogenesis (I), and $\beta$-oxidation (J). Data are presented as means \pm S.D. Statistical differences between experimental groups were determined by the two-tailed $t$ test $(n=6-8$ in each group). $* P<0.05 ; * * P<0.01 ; * * * P<0.001$ vs. MCD group; ${ }^{\#} P<0.05 ;{ }^{\# \# P} P<0.01$;

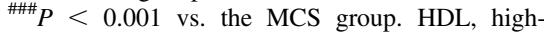
density lipoprotein; LDL, low-density lipoprotein. Original magnification, $\times 20$. Scale bar, $50 \mu \mathrm{m}$. sensitive marker of liver fibrosis. MCD diet-induced upregulation of $\alpha$-smooth muscle actin expression was significantly decreased by GL treatment at the protein level as determined by both immunohistochemical staining (Fig. 2B) and mRNA analysis (Fig. 2C). GL also significantly dampened the expression of fibrogenetic gene mRNAs including transforming growth factor $\beta$-1, tissue inhibitor of metalloproteinases 1 and 2, collagen 1 and 2, and matrix metalloproteinases 2 and 9 (Fig. 2, D-J). These data demonstrate that GL significantly improves NASH-related liver fibrogenesis.

GL Restores NASH-Related Hepatic Lipid Accumulation. In NASH, lipototoxicity caused by accumulating lipids in the liver is a major trigger of liver toxicity (Fuchs and Sanyal, 2012). Therefore, the effect of GL in MCD diet-induced lipid disruption was determined. Compared with the control vehicle-treated MCD group, intraperitoneal injection of $50 \mathrm{mg} / \mathrm{kg}$ GL significantly attenuated MCD diet-induced lipid accumulation in the liver, as revealed by Oil Red O staining (Fig. 3A). Compared with the MCS group, liver TG and TC levels were both significantly increased in the MCD group, and both were significantly decreased by GL treatment (Fig. 3, B and C). In contrast, serum TG and
TC levels were significantly decreased after MCD diet feeding, both of which were also normalized by GL treatment (Fig. 3, D and E). Similarly, GL treatment also normalized MCD diet-induced disruption of serum high-density lipoprotein cholesterol and low-density lipoprotein cholesterol levels (Fig. 3, F and G). These data strongly suggest that GL treatment could improve MCD diet-induced lipid disruption in both the liver and serum.

Systematic lipid homeostasis is maintained by balanced mutual regulation of de novo lipid synthesis and lipid degradation by oxidation and subsequent ketogenesis (Tessari et al., 2009). To examine how GL restores MCD diet-induced dyslipidemia, lipid regulatory pathways were analyzed. Compared with MCS diet-fed mice, MCD diet feeding significantly increased expression of the liver $\mathrm{X}$ receptor and sterol regulatory element-binding transcription factor Srebpl mRNAs and the downstream lipid synthesis gene mRNA, Fas. Although GL treatment decreased the induction of liver $\mathrm{X}$ receptor, Srebp1a, and Srebplc mRNA, it showed no significant effect on the mRNA level of Fas and significantly restored MCD diet-decreased stearoyl-CoA desaturase 1 mRNA, both of which are encoded by two key lipogenic genes 
A

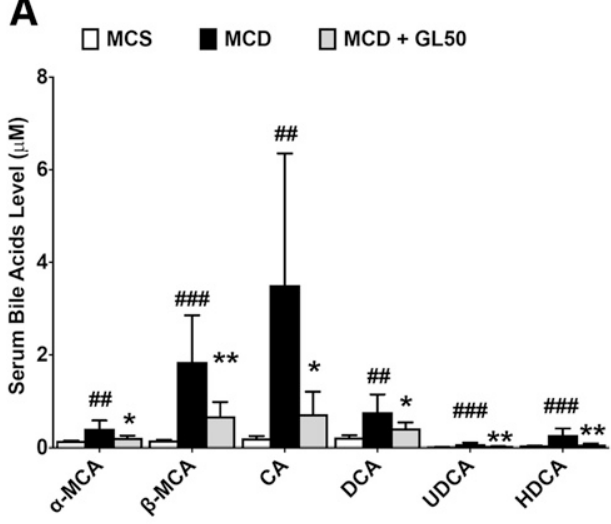

B

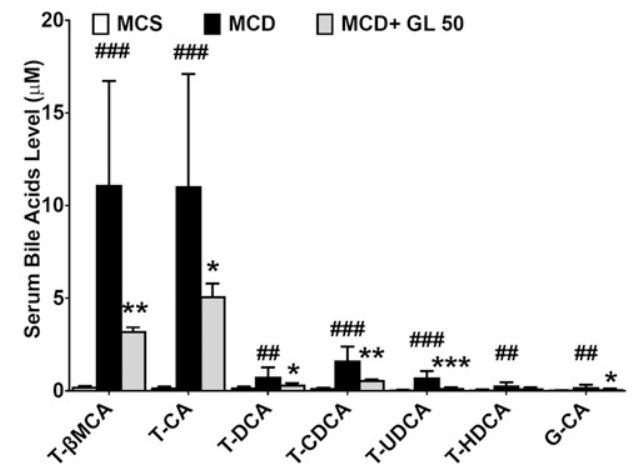

C

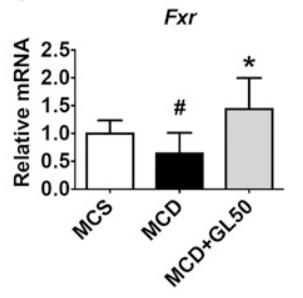

D

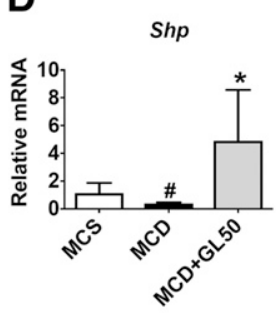

$\mathrm{E}$

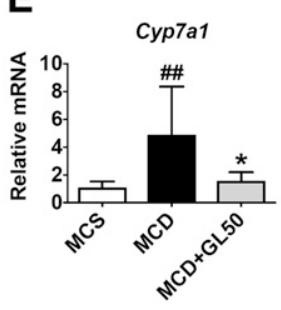

$\mathbf{F}$

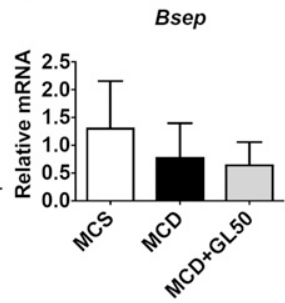

Fig. 4. GL activates liver FXR/SHP/CYP7A1 signaling and rescues MCD-induced bile acid disruption. (A) Serum level of free bile acids $\alpha$-MCA, $\beta$-MCA, CA, DCA, UDCA, and HDCA. (B) Serum level of conjugated bile acids T- $\beta$-MCA, T-CA, T-DCA, T-CDCA, T-UDCA, T-HDCA, and G-CA. (C-F) Hepatic mRNA levels of Fxr (C), Shp (D), Cyp7al (E), and Bsep (F). Data are presented as means \pm S.D. Statistical differences between experimental groups were determined by the two-tailed $t$ test $(n=6-8$ in each group). $* P<0.05$; $* * P<0.01 ; * * * P<0.001$ vs. the MCD group; ${ }^{\#} P<0.05 ;{ }^{\# \#} P<0.01 ;{ }^{\# \# \# P<0.001 \text { vs. the }}$ MCS group. Bsep, bile salt export pump; CDCA, chenodeoxycholic acid; G-CA, glycocholic acid; HDCA, hyodeoxycholic acid; UDCA, ursodeoxycholic acid.
(Fig. 3H). Moreover, GL treatment significantly inhibited MCD dietinduced mRNA upregulation of the fatty acid transport gene mRNAs fatty acid transport proteins 3 and 4, compared with vehicle-treated MCD-fed mice (Fig. 3H). MCD diet feeding did not significantly change the expression of the ketogenesis pathway compared with the MCS dietfed group. In MCD diet-fed mice, GL treatment even significantly decreased acetyl-CoA acetyltransferase 1, hydroxymethylglutaryl-CoA synthase, 3-hydroxy-3-methylglutaryl-CoA lyase, peroxisome proliferator-activated receptor $\alpha$, and Cyp4a14 mRNA levels and showed no significant effect in other mRNA expression involved in ketogenesis and lipid oxidation compared with control vehicle treatment (Fig. 3, I and J). These data indicate that GL may indirectly reduce lipogenesis by suppressing the expression of fatty acid transporters and not through enhancing lipid degradation via ketogenesis and oxidation.

GL Decreases MCD-Induced Bile Acid Accumulation. Bile acids, in accompany with dysregulated lipid metabolism, are commonly accumulated in NASH in both mice and clinical patients. (Bechmann et al., 2013; Gong et al., 2016; Arab et al., 2017). Several species of bile acids may exert direct toxic effects against hepatocytes and serve as danger molecules in triggering NLRP3 inflammasome activation (Gong et al., 2016; Hao et al., 2017). Thus, the effect of GL treatment on serum bile acids was examined under conditions of experimental NASH. MCD diet feeding significantly induced accumulation of free bile acids including $\alpha$-MCA, $\beta$-MCA, CA, deoxycholic acid (DCA), ursodeoxycholic acid, and hyodeoxycholic acid by 2.9-, 12.1-, 18.0-, 3.7-, 7.6-, and 7.2-fold, respectively, all of which were markedly reduced by GL treatment (Fig. 4A). Similarly, GL treatment significantly restored MCD diet-induced serum accumulation of taurine-conjugated bile acids tauro (T)- $\beta$-MCA, T-CA, T-DCA, taurochenodeoxycholic acid, and tauroursodeoxycholic acid and the glycine-conjugated bile acid glycocholic acid (Fig. 4B). FXR activates the expression of SHP-1 that inhibits liver receptor homolog 1 and represses expression of CYP7A1, which catalyzes the rate-limiting step in bile acid biosynthesis (Goodwin et al., 2000). Since this study showed that all tested bile acids including the main primary bile acid (CA) were decreased by GL, GL treatment was inferred to influence the de novo synthesis of bile acids in the liver. Therefore, FXR-SHP-CYP7A1 signaling, the predominant pathway for bile acid synthesis, was analyzed. Hepatic FXR signaling was inhibited and Cyp7al mRNA was upregulated in MCD diet-fed mice compared with MCS diet-fed mice. As expected, GL treatment significantly activated FXR, evidenced by upregulation of Shp mRNA and the downstream suppression of Cyp7al mRNA, whereas Fxr mRNA itself was also significantly increased by GL treatment compared with the vehicle-treated group in the mouse NASH model (Fig. 4, C-E). In contrast, bile salt export pump mRNA was not significantly influenced by GL treatment (Fig. 4F), suggesting a role of GL in inhibiting de novo bile acid synthesis.

To explain how GL could activate FXR in MCD diet-fed livers, the possibility that GL could act as a FXR ligand was explored. In mouse primary hepatocytes and human HepG2 cells in vitro, no significant FXR activation was induced by neither GL nor GA treatment (Supplemental Fig. 2, A and B). In mice in vivo, both single GL injection and multipe GL injection failed to activate FXR (Supplemental Fig. 2C). Consistently, both GL and GA failed to activate FXR, whereas GW4064 (3-(2,6-Dichlorophenyl)-4-(3'-carboxy-2-chlorostilben-4-yl) oxymethyl-5-isopropylisoxazole) induced FXR reporter luciferase by 20-fold as a positive control in HepG2 cells (Supplemental Fig. 2D), excluding GL as a direct FXR activator. These data prompted us to ask whether GL activated FXR only under NASH-related pathologic conditions. Since nuclear factor $\kappa \mathrm{B}(\mathrm{NF}-\kappa \mathrm{B})$ activation, a common pathologic character under NASH conditions, is known to lead to FXR inactivation (Wang et al., 2008), rescue of FXR inactivation induced by NF- $\kappa$ B by GL was examined. LPS treatment slightly but significantly repressed FXR reporter activity, and both GL and GA treatment restored LPS-induced FXR inactivation (Supplemental Fig. 2E). These data suggest that GL may restore bile acid homeostasis via attenuating the loss of FXR signaling induced by inflammatory damage.

GL Inhibits NLRP3 Inflammasome Activation and MetaInflammation in Mice. NLRP3 inflammasome activation is required for the development of fibrosis in NAFLD (Wree et al., 2014a,b; Mridha et al., 2017). Activation of the NLRP3/inflammasome is a two-step process requiring a priming step (signal 1) induced by binding of 

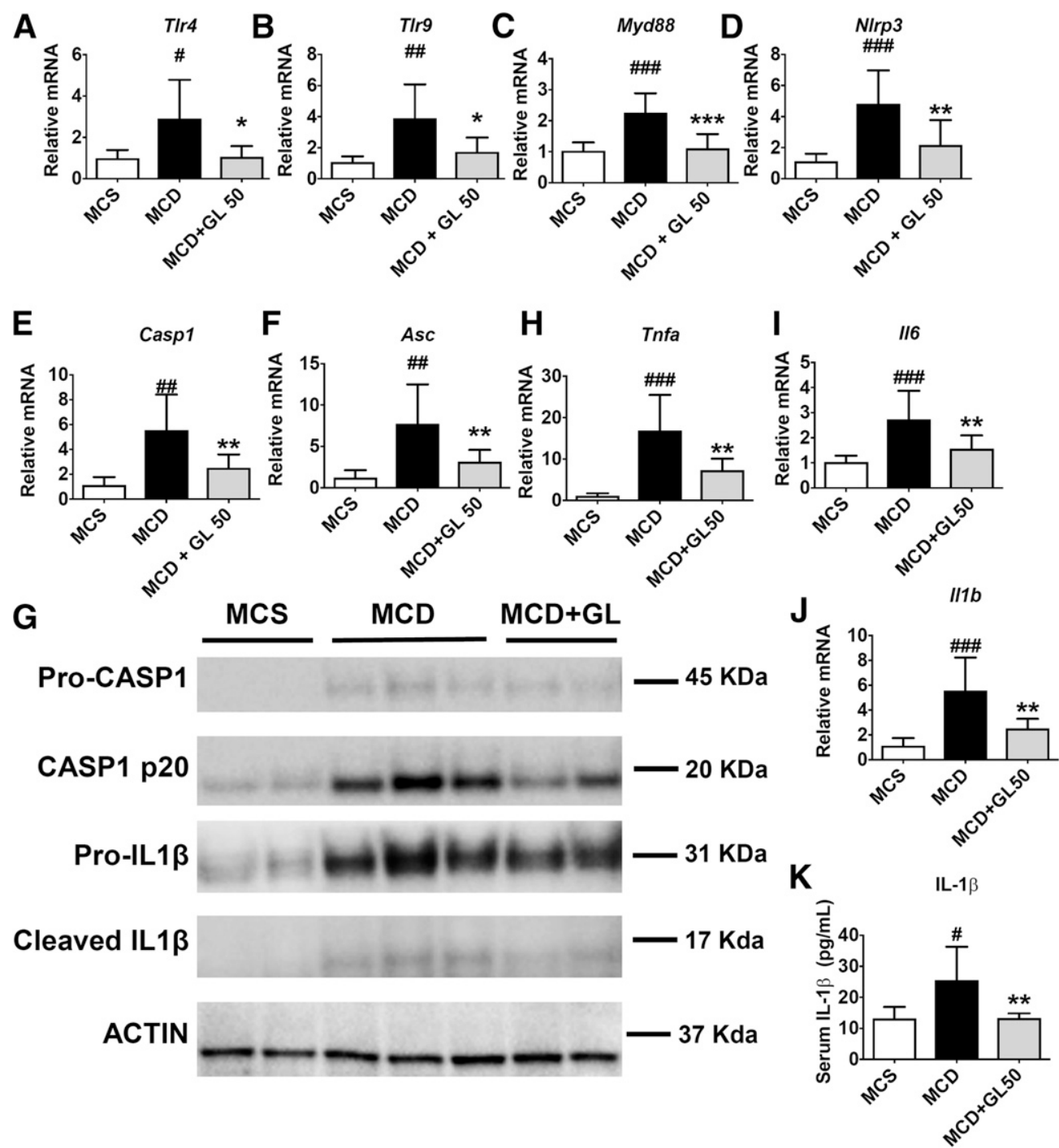

Fig. 5. GL significantly reduces MCD-induced TLR/NLRP3 inflammasome activation and related meta-inflammation. (A-C) mRNA levels of $T l r 4$ (A), Tlr9 (B), and Myd88 (C) in the liver. (D-F) mRNA levels of Nlrp3 (D), Casp1 (E), and Asc (F) in the liver. (G) Western blot analysis of pro-CASP1, cleaved CASP1, pro-IL-1 $\beta$, and cleaved IL-1 $\beta$ in the liver. (H-J) mRNA levels of proinflammatory cytokines Tnfa $(\mathrm{H}), I l 6(\mathrm{I})$, and $I l l b$ (J) in the liver. (K) ELISA analysis of serum IL-1 $\beta$ levels in mice. Data are presented as means \pm S.D. Statistical differences between experimental groups were determined by the two-tailed $t$ test $(n=6-8$ in each group). $* P<0.05 ; * * P<$ $0.01 ; * * * P<0.001$ vs. the MCD group; ${ }^{\#} P<0.05 ;{ }^{\# \#} P<0.01 ;{ }^{\# \# \#} P<0.001$ vs. the MCS group. Asc, apoptosis-associated speck-like protein containing a caspase recruitment domain; ELISA, enzyme-linked immunosorbent assay; Myd88, myeloid differentiation primary response 88

damage-associated molecular patterns (DAMPs) or pathogen-associated molecular patterns to receptors such as Toll-like receptor (TLR) to increase the transcription of NLRP3 and pro-IL-1 $\beta$, followed by an activation step (signal 2) induced by the recruitment of apoptosisassociated speck-like protein containing a caspase recruitment domain to activate the NLRP3 inflammasome to convert pro-CASP1 into active CASP1, which in turn processes pro-IL- $1 \beta$ into mature IL-1 $\beta$ (Guo et al., 2015). Since GL is a known inhibitor of the NLRP3 inflammasome (Honda et al., 2014), we tested whether GL inhibited activation of the NLRP3 inflammasome in the NASH model. Compared with MCS diet-fed mice, MCD diet feeding induced a significant 2- to 4-fold increase in upstream $T l r 4, T l r 9$, and myeloid differentiation primary response 88 mRNAs, all of which were normalized by GL treatment (Fig. 5, A-C). Similarly, the MCD diet induced Casp1, Nlrp3, and apoptosis-associated speck-like protein containing a caspase recruitment domain mRNAs by 5.5-, 4.8-, and 7.7-fold, respectively, which were significantly reduced by GL treatment (Fig. 5, D-F). These data suggest that GL could dampen the signal 1 activation of NLRP3/inflammasome in MCD diet-fed mice. Western blot analysis was performed to test the protein levels of pro-CASP1, active CASP1, pro-IL- $1 \beta$, and active IL- $1 \beta$. The MCD diet induced upregulation of signals 1 and 2 compared with MCS diet treatment. GL treatment decreased the protein expression of pro-CASP1 and pro-IL- $1 \beta$ (Fig. 5G), confirming GL's effect in inhibiting signal 1 of NLRP3 inflammasome activation. Furthermore, GL treatment was also found to inhibit the MCD diet-induced upregulation of active CASP1 (p20) and cleaved IL-1 $\beta$ (Fig. $5 \mathrm{G})$. In addition, GL significantly inhibited the upregulation of downstream proinflammatory cytokine mRNAs Tnfa, Il6, and $I l l b$ (Fig. 5, H-J) and serum release of IL- $1 \beta$ protein (Fig. $5 \mathrm{~K}$ ), demonstrating a significant improvement in NASH-related meta-inflammation by GL. These data demonstrate that GL treatment could dampen MCD diet-induced TLR/ NLRP3 inflammasome activation and the related meta-inflammation.

Since previous reports and our results indicate that bile acids accumulate in NASH and may be the main trigger for activation of the NLRP3 inflammsome (Arab et al., 2017; Hao et al., 2017), we explored the possibility of whether GL could directly inhibit bile acid-induced NLRP3 inflammasome activation. DCA was shown to induce NLRP3 
A

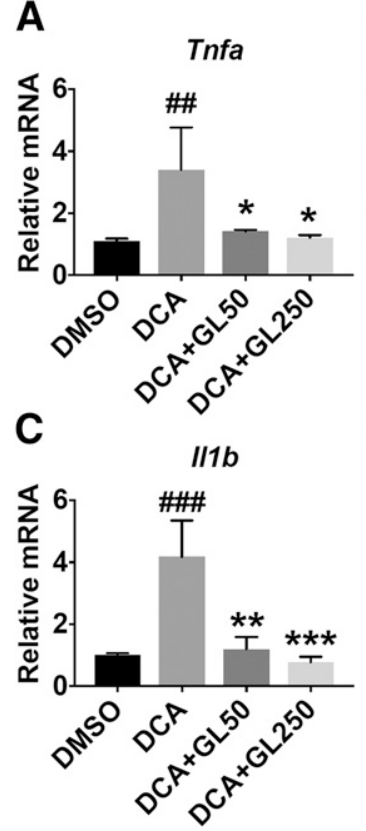

$E$

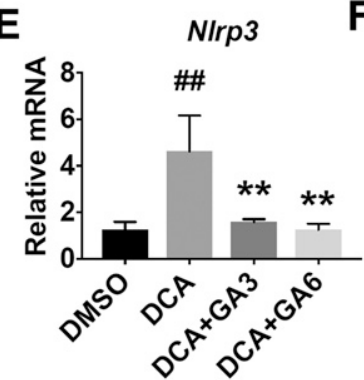

B

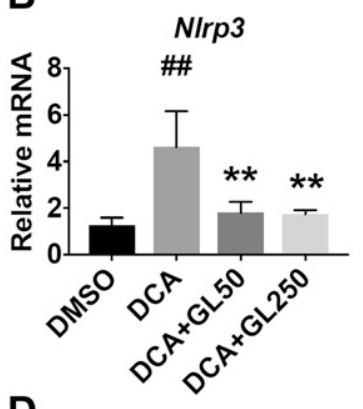

D

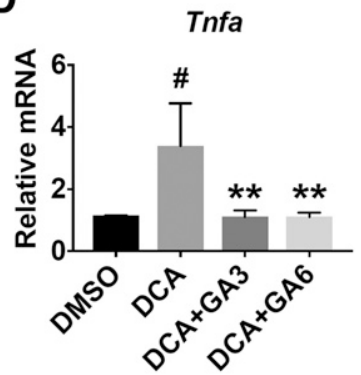

$\mathbf{F}$

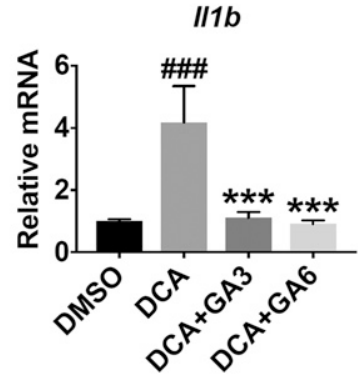

Fig. 6. Both GL and GA significantly inhibit DCA-induced Tnfa, Nlrp3, and $I l 1 b$ mRNA. (A-C) Effect of GL in DCA-induced mRNA of Tnfa (A), Nlrp3 (B), and $I l 1 b$ (C) mRNAs in Raw 264.7 cells. (D-F) Effect of GA in DCA-induced Tnfa (D), Nlrp3 (E), and $I 11 b$ (F) mRNAs in Raw 264.7 cells. Data are presented as means \pm S.D. Statistical differences were determined by one-way analysis of variance followed by the Dunnett multiple-comparisons test among multiple-group comparisons ( $n=3$ per group). Raw 264.7 cells were pretreated with $0.1 \%$ DMSO, GL, or GA for 30 minutes and then treated with $200 \mu \mathrm{M}$ DCA for an additional 4 hours to further perform mRNA analysis. $* P<0.05$; *** $P<0.01$; *** $P<0.001$ vs. the

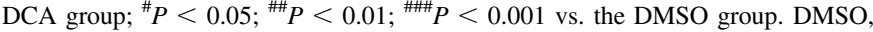
dimethylsulfoxide.

inflammasome activation (Hao et al., 2017) and was thus used as a model to test GL's effect in inhibiting bile acid-induced NLRP3 inflammasome activation. In Raw 264.7 cells, both GL and GA significantly decreased DCA-induced upregulation of Tnfa, Nlrp3, and $I l l b$ mRNAs (Fig. 6). These data demonstrate that GL and its metabolite GA could directly suppress DCA-induced NLRP3 inflammasome activation in vitro, which at least partially explains GL's role in dampening MCD diet-induced NLRP3 inflammasome activation in vivo.

GA, the Active Metabolite of GL, Improves MCD Diet-Induced Liver Injury. Structurally, GL is a glycoside that is hydrolyzed into its metabolite GA by intestinal bacteria after oral administration (Akao et al., 1994; Takeda et al., 1996). To test whether GA influences NASH, mice were treated with the MCD diet for 6 weeks and then either intraperitoneally injected with GA $(30 \mathrm{mg} / \mathrm{kg})$ or intragastrically administered GL by gavage $(50 \mathrm{mg} / \mathrm{kg}$ ) for the last 3 weeks (Fig. 7A). GA as well as GL gavage did not significantly change the body weight, liver weight, and liver index (Supplemental Fig. 3). GA significantly decreased the MCD diet-induced increase in ALT and AST levels

(Fig. 7, B and C). Similarly, GL gavage at a dose of $50 \mathrm{mg} / \mathrm{kg}$ also significantly ameliorated the MCD diet-induced increase in serum ALT and AST levels (Fig. 7, D and E). Histologic analysis showed that $30 \mathrm{mg} /$ $\mathrm{kg}$ GA ameliorated MCD diet-induced hepatic steatosis (Fig. 7, F and G). Similarly, GL gavage at a dose of $50 \mathrm{mg} / \mathrm{kg}$ improved liver lipid overloading (Fig. 7, H and I). These results indicate that GA may be required for the hepatoprotective effects of GL for the treatment of NASH.

\section{Discussion}

GL and its active metabolite GA have been demonstrated to be hepatoprotective; however, they have not yet been examined for their activities toward NASH. This study shows that GL, via its activate metabolite GA, could decrease MCD diet-induced NASH, as revealed by improved hepatic steatosis, inflammation, and fibrosis. Mechanistically, GL may restore NASH-induced dysregulation of bile acids and lipids and the resultant meta-inflammation.

Many factors such as cholesterol crystal (Duewell et al., 2010), ceramides (Chaurasia and Summers, 2015), and secondary bile acids (Hao et al., 2017) are known to accumulate in NASH livers and could act as DAMPs to induced NLRP3 inflammasome activation. Activation of the NLRP3 inflammasome results in the release of IL- $1 \beta$, together with other proinflammatory cytokines such as $\mathrm{TNF} \alpha$, which triggers an inflammatory cascade that ultimately promotes pathologic development of NASH. Thus, accumulated DAMPs, via activation of the NLRP3 inflammasome and other inflammatory pathways, may represent core pathologic triggers in NASH development. In our study using the MCD diet-induced NASH model, GL was shown to inhibit NLRP3 inflammasome activation accompanied by restoring abnormal serum bile acid accumulation, and GL could normalize the dysregulated lipids and bile acids and thereby reduce meta-inflammation. Since several bile acids are known to activate NLRP3/inflammasome signaling, GL may inhibit the NLRP3 inflammasome via the regulation of FXR signaling. However, GL did not activate FXR in normal mice in vivo or hepatocytes in vitro and failed to induce FXR reporter luciferase activity, indicating that GL is not a direct FXR activator. FXR is known to have reciprocal regulation with NF- $\kappa \mathrm{B}$ activation (Wang et al., 2008). FXR signaling is reduced under excessive inflammation, resulting in the accumulation of bile acids and dysregulated lipid catabolism, which reciprocally triggers further inflammation (Bechmann et al., 2013). Although the exact mechanism warrants further clarification, this study strongly indicates that GL and GA may target FXR-mediated meta-inflammation in the treatment of NASH. Our data agree with a previous report showing that GL induces SHP expression and inhibits Cyp7al expression in a lithocholic acidinduced cholestatic liver injury model (Wang et al., 2012). In addition, because the accumulation of bile acids may directly damage hepatocytes (Gumpricht et al., 2005), the observation that GL can restore bile acid homeostasis suggests that GL may be particularly suitable for treating NASH that has the feature of cholestasis.

In addition to restoring lipid and bile acid homeostasis, GL could also directly inhibit DCA-induced NLRP3 inflammasome activation, suggesting that it may target multiple nodes in treating NASH. In line with our findings, GL was previously shown to inhibit NLRP3 inflammasome activation induced by diverse DAMPs or pathogen-associated molecular patterns in bone marrow-derived macrophages (Honda et al., 2014). MCC950 ( $N$-[[(1,2,3,5,6,7-hexahydro-s-indacen-4-yl)amino] carbonyl]-4-(1-hydroxy-1-methylethyl)-2-furansulfonamide sodium salt), an NLRP3 selective inhibitor, was recently demonstrated to improve NAFLD pathology and fibrosis (Mridha et al., 2017), suggesting that the NLRP3 inflammasome might be a potential target for NASH therapy. Moreover, it was found that MCD 
A

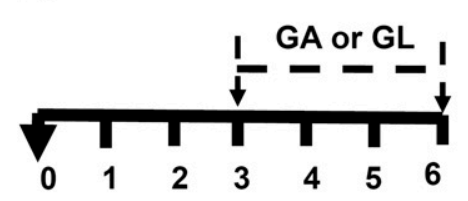

MCD Feeding Time (W, Week)

B

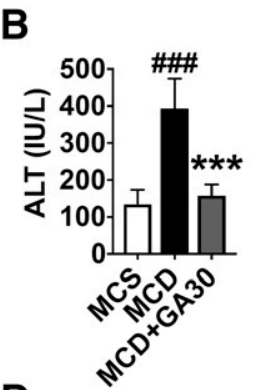

D

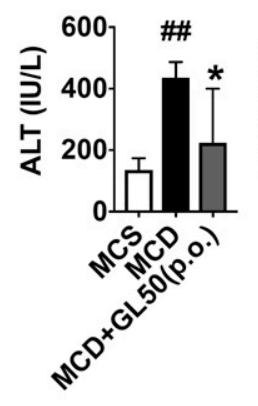

C

E

\section{F MCD}
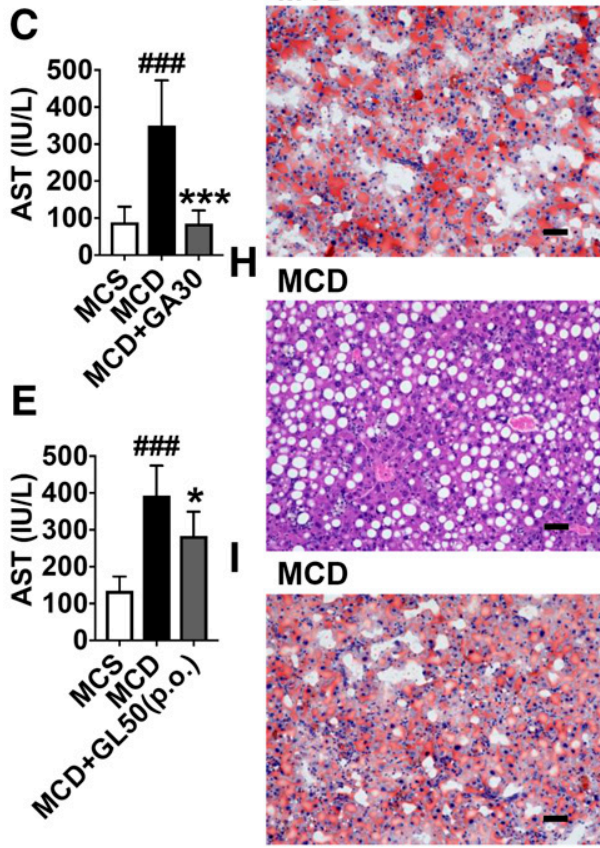

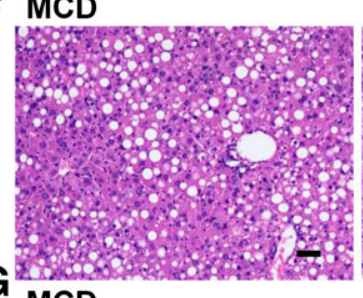

MCD

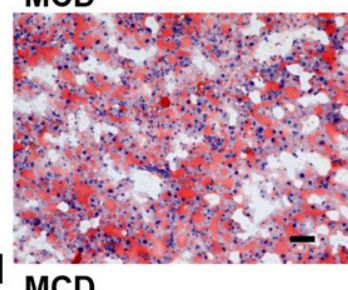

$M C D+G A 30$

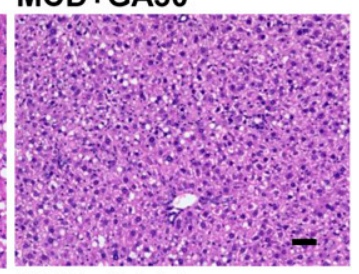

$M C D+G A 30$

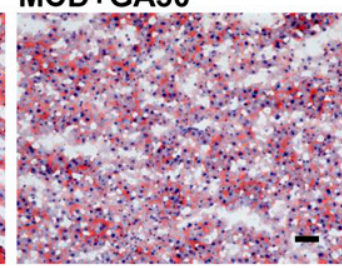

MCD+GL50 (P.O.)

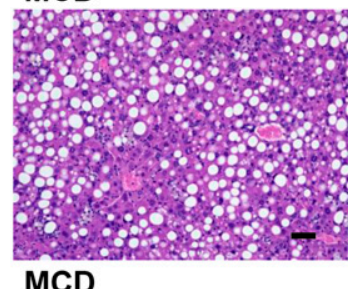

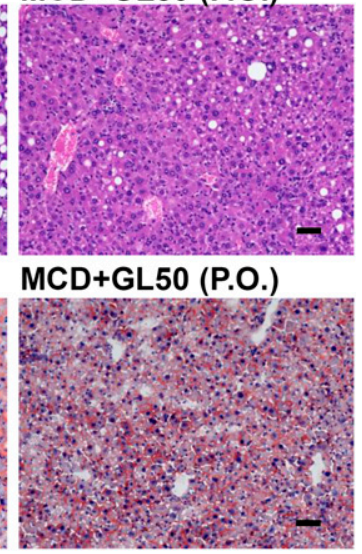

Fig. 7. GL's active metabolite, GA, significantly reduces MCD-induced liver injury. (A) Mouse experiment procedure scheme. (B and C) Levels of serum ALT (B) and AST (C) in saline- or GA-treated mice. (D and E) Levels of serum ALT (D) and AST (E) in saline- or GL gavage-treated mice. ( $F$ and $G$ ) Representative $H \& E$ staining $(F)$ and Oil Red $\mathrm{O}$ staining $(\mathrm{G})$ for livers from the MCD group and the $\mathrm{MCD}+\mathrm{GA} 30$ group. ( $\mathrm{H}$ and $\mathrm{I}$ ) Representative $\mathrm{H} \& \mathrm{E}$ staining $(\mathrm{H})$ and Oil Red $\mathrm{O}$ staining (I) for livers from the MCD group and MCD+GL50 (p.o.) group. Data are presented as means \pm S.D. Statistical differences between experimental groups were determined by the two-tailed $t$ test ( $n=5$ in each group). $* P<0.05$ and $* * * P<0.001$ vs. the MCD group; ${ }^{\# \#} P<0.01$

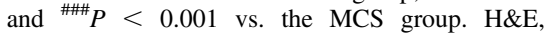
hematoxylin and eosin. Original magnification, $20 \times$; Scale bar, $50 \mu \mathrm{m}$. diet-induced steatosis sensitized cholestatic liver injury and dysregulated bile acid synthesis and transport (Lionarons et al., 2016). Our results, together with these previous findings, strongly indicate that dysregulated metabolism of lipids and bile acids and the resultant meta-inflammation is a pivotal event in NASH development.

GL administered by the oral route shows extremely low bioavailability (approximately 1\%) and most GL by oral intake is hydrolyzed into GA in the gut by intestinal bacteria, which is then absorbed and elicits its pharmacological effects (Nose et al., 1994; Yamamura et al., 1995; Takeda et al., 1996). In line with this point, GL gavage and direct GA intraperitoneal injection could both combat experimental NASH, suggesting that the effect of GL against MCD diet-induced NASH is largely through its primary metabolite GA. In contrast, GL (but not GA) contributes to the hepatoprotective effect of GL in ameliorating acetaminophen-induced liver injury (Yan et al., 2016). Thus, it is important to note that although both GL and GA have efficacy in various types of hepatic injury, the exact effects and thereby precise clinical applications of these agents need to be carefully considered.

In summary, the herbal medicine-derived compound GL may be a therapeutic option for treating NASH, particularly that harbors the symptom of cholestasis. Mechanistically, GL and/or GA may target bile acid-mediated meta-inflammation and block the reciprocal FXR-NLRP3 inflammasome pathway. All of the data obtained from our MCD dietinduced NASH model demonstrate that GL and GA may be potentially effective against NASH. However, it is important to note that the MCD diet-induced NASH model, despite the presented liver steatosis and inflammation, does not show obesity or insulin resistance or mimic the aberrant increase of serum lipids, all of which are common pathophysiological characteristics of human NASH (Hebbard and George, 2011).
Thus, studies that use a long-term high-fat and/or high-sugar diet feeding model would help further confirm the translational potential of GL and GA in combating metabolic syndrome-related NASH.

\section{Acknowledgments}

We thank Grace L. Guo for providing the PGL4-Shp-TK firefly luciferase construct and human FXR expression plasmid.

\section{Authorship Contributions}

Participated in research design: Yan, H. Wang, G. Wang, Gonzalez, Hao. Conducted experiments: Yan, H. Wang, Q. Wang, Takahashi, Yagai, Li.

Contributed new reagents or analytic tools: Cao, Krausz.

Performed data analysis: Yan, $\mathrm{H}$. Wang.

Wrote or contributed to the writing of the manuscript: Yan, Gonzalez, Hao.

\section{References}

Akao T, Hayashi T, Kobashi K, Kanaoka M, Kato H, Kobayashi M, Takeda S, and Oyama T (1994) Intestinal bacterial hydrolysis is indispensable to absorption of 18 beta-glycyrrhetic acid after oral administration of glycyrrhizin in rats. J Pharm Pharmacol 46:135-137.

Arab JP, Karpen SJ, Dawson PA, Arrese M, and Trauner M (2017) Bile acids and nonalcoholic fatty liver disease: molecular insights and therapeutic perspectives. Hepatology 65:350-362. Bechmann LP, Kocabayoglu P, Sowa JP, Sydor S, Best J, Schlattjan M, Beilfuss A, Schmitt J, Hannivoort RA, Kilicarslan A, et al. (2013) Free fatty acids repress small heterodimer partner (SHP) activation and adiponectin counteracts bile acid-induced liver injury in superobese patients with nonalcoholic steatohepatitis. Hepatology 57:1394-1406.

Chaurasia B and Summers SA (2015) Ceramides - lipotoxic inducers of metabolic disorders. Trends Endocrinol Metab 26:538-550.

Duewell P, Kono H, Rayner KJ, Sirois CM, Vladimer G, Bauernfeind FG, Abela GS, Franchi L, Nuñez G, Schnurr M, et al. (2010) NLRP3 inflammasomes are required for atherogenesis and activated by cholesterol crystals. Nature 464:1357-1361.

Feldstein AE, Canbay A, Angulo P, Taniai M, Burgart LJ, Lindor KD, and Gores GJ (2003) Hepatocyte apoptosis and fas expression are prominent features of human nonalcoholic steatohepatitis. Gastroenterology 125:437-443.

Fuchs M and Sanyal AJ (2012) Lipotoxicity in NASH. J Hepatol 56:291-293. 
Gong Z, Zhou J, Zhao S, Tian C, Wang P, Xu C, Chen Y, Cai W, and Wu J (2016) Chenodeoxycholic acid activates NLRP3 inflammasome and contributes to cholestatic liver fibrosis. Oncotarget 7:83951-83963.

Goodwin B, Jones SA, Price RR, Watson MA, McKee DD, Moore LB, Galardi C, Wilson JG, Lewis MC, Roth ME, et al. (2000) A regulatory cascade of the nuclear receptors FXR, SHP-1, and LRH-1 represses bile acid biosynthesis. Mol Cell 6:517-526.

Gumpricht E, Dahl R, Devereaux MW, and Sokol RJ (2005) Licorice compounds glycyrrhizin and 18beta-glycyrrhetinic acid are potent modulators of bile acid-induced cytotoxicity in rat hepatocytes. J Biol Chem 280:10556-10563.

Guo H, Callaway JB, and Ting JP (2015) Inflammasomes: mechanism of action, role in disease, and therapeutics. Nat Med 21:677-687.

Hao H, Cao L, Jiang C, Che Y, Zhang S, Takahashi S, Wang G, and Gonzalez FJ (2017) Farnesoid $\mathrm{X}$ receptor regulation of the NLRP3 inflammasome underlies cholestasis-associated sepsis. Cell Metab 25:856-867.e5.

Hebbard L and George J (2011) Animal models of nonalcoholic fatty liver disease. Nat Rev Gastroenterol Hepatol 8:35-44.

Honda H, Nagai Y, Matsunaga T, Okamoto N, Watanabe Y, Tsuneyama K, Hayashi H, Fujii I, Ikutani M, Hirai Y, et al. (2014) Isoliquiritigenin is a potent inhibitor of NLRP3 inflammasome activation and diet-induced adipose tissue inflammation. J Leukoc Biol 96:1087-1100.

Jadeja R, Devkar RV, and Nammi S (2014) Herbal medicines for the treatment of nonalcoholic steatohepatitis: current scenario and future prospects. Evid Based Complement Alternat Med 2014:648308.

Leist M, Gantner F, Bohlinger I, Germann PG, Tiegs G, and Wendel A (1994) Murine hepatocyte apoptosis induced in vitro and in vivo by TNF-alpha requires transcriptional arrest. $J$ Immunol 153:1778-1788.

Li JY, Cao HY, Liu P, Cheng GH, and Sun MY (2014) Glycyrrhizic acid in the treatment of liver diseases: literature review. BioMed Res Int 2014:872139.

Lionarons DA, Heger M, van Golen RF, Alles LK, van der Mark VA, Kloek JJ, de Waart DR, Marsman HA, Rusch H, Verheij J, et al. (2016) Simple steatosis sensitizes cholestatic rats to liver injury and dysregulates bile salt synthesis and transport. Sci Rep 6:31829.

Mridha AR, Wree A, Robertson AAB, Yeh MM, Johnson CD, Van Rooyen DM, Haczeyni F, Teoh NC, Savard C, Ioannou GN, et al. (2017) NLRP3 inflammasome blockade reduces liver inflammation and fibrosis in experimental NASH in mice. J Hepatol 66:1037-1046.

Nose M, Ito M, Kamimura K, Shimizu M, and Ogihara Y (1994) A comparison of the antihepatotoxic activity between glycyrrhizin and glycyrrhetinic acid. Planta Med 60:136-139.

Osborn O and Olefsky JM (2012) The cellular and signaling networks linking the immune system and metabolism in disease. Nat Med 18:363-374.

Schoemaker MH, Gommans WM, Conde de la Rosa L, Homan M, Klok P, Trautwein C, van Goor H, Poelstra K, Haisma HJ, Jansen PLM, et al. (2003) Resistance of rat hepatocytes against bile acid-induced apoptosis in cholestatic liver injury is due to nuclear factor-kappa B activation. $J$ Hepatol 39:153-161.

Takeda S, Ishthara K, Wakui Y, Amagaya S, Maruno M, Akao T, and Kobashi K (1996) Bioavailability study of glycyrrhetic acid after oral administration of glycyrrhizin in rats; relevance to the intestinal bacterial hydrolysis. J Pharm Pharmacol 48:902-905.

Tessari P, Coracina A, Cosma A, and Tiengo A (2009) Hepatic lipid metabolism and non-alcoholic fatty liver disease. Nutr Metab Cardiovasc Dis 19:291-302.

Wang CY, Mayo MW, and Baldwin AS, Jr (1996) TNF- and cancer therapy-induced apoptosis: potentiation by inhibition of NF-kappaB. Science 274:784-787.

Wang H, Fang ZZ, Meng R, Cao YF, Tanaka N, Krausz KW, and Gonzalez FJ (2017) Glycyrrhizin and glycyrrhetinic acid inhibits alpha-naphthyl isothiocyanate-induced liver injury and bile acid cycle disruption. Toxicology 386:133-142.
Wang YD, Chen WD, Wang M, Yu D, Forman BM, and Huang W (2008) Farnesoid X receptor antagonizes nuclear factor kappaB in hepatic inflammatory response. Hepatology 48:1632-1643.

Wang YG, Zhou JM, Ma ZC, Li H, Liang QD, Tan HL, Xiao CR, Zhang BL, and Gao Y (2012) Pregnane $\mathrm{X}$ receptor mediated-transcription regulation of CYP3A by glycyrrhizin: a possible mechanism for its hepatoprotective property against lithocholic acid-induced injury. Chem Biol Interact 200:11-20.

Wong CR, Nguyen MH, and Lim JK (2016) Hepatocellular carcinoma in patients with nonalcoholic fatty liver disease. World J Gastroenterol 22:8294-8303.

Wong RJ, Cheung R, and Ahmed A (2014) Nonalcoholic steatohepatitis is the most rapidly growing indication for liver transplantation in patients with hepatocellular carcinoma in the U.S Hepatology 59:2188-2195.

Wree A, Eguchi A, McGeough MD, Pena CA, Johnson CD, Canbay A, Hoffman HM, and Feldstein AE (2014a) NLRP3 inflammasome activation results in hepatocyte pyroptosis, liver inflammation, and fibrosis in mice. Hepatology 59:898-910.

Wree A, McGeough MD, Peña CA, Schlattjan M, Li H, Inzaugarat ME, Messer K, Canbay A, Hoffman HM, and Feldstein AE (2014b) NLRP3 inflammasome activation is required for fibrosis development in NAFLD. J Mol Med (Berl) 92:1069-1082.

Wu X, Zhang L, Gurley E, Studer E, Shang J, Wang T, Wang C, Yan M, Jiang Z, Hylemon PB, et al. (2008) Prevention of free fatty acid-induced hepatic lipotoxicity by 18beta-glycyrrhetinic acid through lysosomal and mitochondrial pathways. Hepatology 47:1905-1915.

Xie G, Wang X, Huang F, Zhao A, Chen W, Yan J, Zhang Y, Lei S, Ge K, Zheng X, et al. (2016) Dysregulated hepatic bile acids collaboratively promote liver carcinogenesis. Int J Cancer 139: $1764-1775$.

Yamamura Y, Santa T, Kotaki H, Uchino K, Sawada Y, and Iga T (1995) Administration-route dependency of absorption of glycyrrhizin in rats: intraperitoneal administration dramatically enhanced bioavailability. Biol Pharm Bull 18:337-341.

Yan T, Wang H, Zhao M, Yagai T, Chai Y, Krausz KW, Xie C, Cheng X, Zhang J, Che Y, et al (2016) Glycyrrhizin protects against acetaminophen-induced acute liver injury via alleviating tumor necrosis factor $\alpha$-mediated apoptosis. Drug Metab Dispos 44:720-731.

Younossi ZM, Koenig AB, Abdelatif D, Fazel Y, Henry L, and Wymer M (2016) Global epidemiology of nonalcoholic fatty liver disease-meta-analytic assessment of prevalence, incidence, and outcomes. Hepatology 64:73-84.

Zhang L and Schuppan D (2014) Traditional Chinese Medicine (TCM) for fibrotic liver disease: hope and hype. J Hepatol 61:166-168.

Zhou L, Song Y, Zhao J, Qin H, Zhang G, Zhou Y, and Wu X (2016) Monoammonium glycyrrhizinate protects rifampicin- and isoniazid-induced hepatotoxicity via regulating the expression of transporter Mrp2, Ntcp, and Oatp1a4 in liver. Pharm Biol 54:931-937.

Zoller $\mathrm{H}$ and Tilg $\mathrm{H}$ (2016) Nonalcoholic fatty liver disease and hepatocellular carcinoma. Metabolism 65:1151-1160.

Address correspondence to: Frank J. Gonzalez, Laboratory of Metabolism, Center for Cancer Research, National Institutes of Health National Cancer Institute, Bethesda, MD 20892. E-mail: gonzalef@mail.nih.gov; or Haiping Hao, State Key Laboratory of Natural Medicines, Key Laboratory of Drug Metabolism and Pharmacokinetics, China Pharmaceutical University, No. 24, Tongjiaxiang, Nanjing 210009, China. E-mail: haipinghao@cpu.edu.cn 